\title{
A New Differential Light Scattering Photometer
}

\author{
Tomo-o Oyama, Kohzoh ShIOKAWA, and Koumei BABA \\ Department of Applied Science, Faculty of Engineering, \\ Kyushu University, Hakozaki, Higashi-ku, \\ Fukuoka 812, Japan.
}

(Received July 26, 1980)

\begin{abstract}
The relation between the radius of gyration and the ratio $\left[\left(R_{\theta}-R_{\pi-\theta}\right) /\left(R_{\theta}+\right.\right.$ $\left.\left.R_{\pi-\theta}\right)\right]_{\mathrm{C}=0}$, where $R_{\theta}$. is the scattering intensity at scattering angle $\theta$ and $C$ is the concentration, was derived. In view of the fact that $\left(R_{\theta}-R_{\pi-\theta}\right)$ is small for the small radius of gyration, a new light scattering photometer, allowing the direct determination of $\left(R_{\theta}-R_{\pi-\theta}\right)$, was constructed. It was experimentally confirmed that this photometer can be applied to the estimation of the radius of gyration for polystyrene down to $5 \mathrm{~nm}$. Problems in instrumentation and performance are discussed.
\end{abstract}

KEY WORDS Light Scattering / Scattering Photometer / Radius of Gyration / Differential Method / Polystyrene /

The mean square radius of gyration $\left\langle S^{2}\right\rangle$ is an important characteristic quantity of a polymer chain. This quantity can be determined in various types of scattering experiments. Light, X-rays and neutrons are used as the wave source of scattering. Among these three types of scattering experiments, light scattering (LS) using a laser beam as the light source is the most familiar method for measuring $\left\langle S^{2}\right\rangle$ of polymer solutions. However, the range of $q(\equiv(4 \pi / \lambda) \sin \theta / 2 ; \lambda$, wave length; $\theta$, scattering angle) of LS is often too small to estimate $\left\langle S^{2}\right\rangle$ of globular or low-molecular-weight polymers. For this reason, the range of $\left\langle S^{2}\right\rangle$ which can be measured with a high accuracy by using a conventional LS instrument has been limited to that of above about $15-20 \mathrm{~nm}(\sim \lambda / 20)$.

In this study, a new LS photometer is constructed in order to overcome this limitation and its applicability for the determination of small $\left\langle S^{2}\right\rangle$ is demonstrated on polystyrene samples.

\section{THEORY}

As well known, $R_{\theta}$, the scattering intensity from a solution at scattering angle $\theta$, is given by

$$
R_{\theta}=K M H(\theta, C) C
$$

where $K$ is a constant, $M$ is the molecular weight of the solute molecule, $C$ is the concentration and $H(\theta$, $C$ ) is the scattering function. Now, we consider only infinitely dilute solutions. In such cases, $R_{\theta}$ can be represented by

$$
R_{\theta}=K M P(\theta) C
$$

where $P(\theta)=H(\theta, C)_{\mathrm{C} \rightarrow 0}$. When $q r_{i j} \ll 1$ for all $i$ and $j\left(r_{i j}\right.$; the distance between the $i$ th and the $j$ th segments in a polymer chain), $P(\theta)$ can be expanded as follows, ${ }^{1}$

$P(\theta)=1-\rho \sin ^{2}\left(\frac{\theta}{2}\right)+\frac{3}{20} \beta_{2} \rho^{2} \sin ^{4}\left(\frac{\theta}{2}\right)+O\left(\rho^{3}\right)$

where

$$
\rho=\frac{16 \pi^{2}}{3} \frac{\left\langle S^{2}\right\rangle}{\lambda^{2}}
$$

and $\left\langle S^{2}\right\rangle$ is the mean-square radius of gyration of the solute polymer. $\beta_{2}$ denotes

$$
\sum_{i j}\left\langle r_{i j}^{4}\right\rangle /\left(\sum_{i j}\left\langle r_{i j}^{2}\right\rangle\right)^{2}
$$

and depends on the shape of the polymer chain. The value of $\beta_{2}$ is 5 for the Gaussian chain and is the same order for real chains.

From eq 2 and 3, the next equation is derived as

$$
\frac{R_{\theta}-R_{\pi-\theta}}{R_{\theta}+R_{\pi-\theta}}=\frac{\cos \theta}{2} \rho_{\mathrm{app}}
$$


where

$$
\rho_{\mathrm{app}}=\rho\left[1+\left(\frac{1}{2}-\frac{3}{20} \beta_{2}\right) \rho+O\left(\rho^{2}\right)\right]
$$

According to eq $5, \rho_{\text {app }}$ is evaluated using the left side quantity which can be measured without any instrument calibration for absolute values of $R_{\theta}$ and $R_{\pi-\theta}$. The numerator in this quantity is the difference between the scattering intensities at the mutually supplementary angles and is the decisive term for determing $\rho_{\text {app }}$. In the case of low-molecularweight polymers, this difference is very small, so that no sufficiently high accuracy and sensitivity for determining $\rho_{\text {app }}$ can be attained so long as $R_{\theta}$ and $R_{\pi-\theta}$ are separately measured. If this difference can be directly measured with high accuracy, the measurable range of $\rho_{\text {app }}\left(\sim\left\langle S^{2}\right\rangle\right)$ should be improved. In the section on instrumentation, a new LS photometer is presented, and allows one directly to determine this difference by introducing a new optical system and an electric device.

For the Guassian chain $\left(\beta_{2}=5\right), \rho_{\text {app }}$ is approximated by neglecting $O\left(\rho^{2}\right)$ in eq 6 as

$$
\rho_{\mathrm{app}}=\rho\left(1-\frac{\rho}{4}\right)
$$

The plots of exact (calculated from the left side of eq 5 by using the Debye's scattering function) and approximated (eq 7) values of $\rho_{\text {app }}$ against $\left\langle S^{2}\right\rangle^{1 / 2} / \lambda$

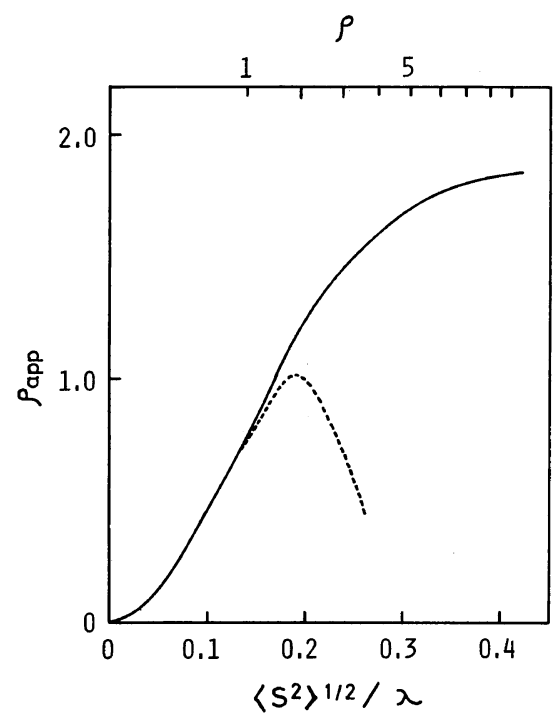

Figure 1. The relation of $\rho_{\text {app }}$ to $\left\langle S^{2}\right\rangle^{1 / 2} / \lambda$ and $\rho$ : solid line, exact Debye's function; dotted line, eq 7 . (and $\rho$ ) are shown in Figure 1. The exact value of $\rho_{\text {app }}$ reaches asymptotically 2 as $\rho \rightarrow \infty$. If $\rho$ $\sin ^{2} \theta / 2<(10 / 3), P(\theta)$ of real chains can be approximated by that of the Gaussian chain. ${ }^{2}$ Thus, $\left\langle S^{2}\right\rangle^{1 / 2}$ is calculated by using eq 7 in good approximation when $\rho_{\text {app }}<0.6$.

\section{INSTRUMENTATION}

The block diagram of the new LS photometer is shown in Figure 2. The laser beam emitted from an

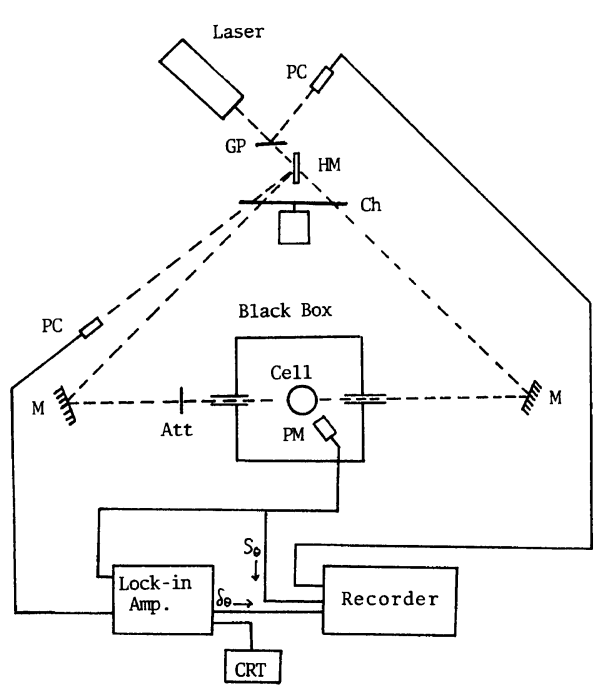

Figure 2. Block diagram of optical and electrical systems: solid line, electric signal path; dotted line, light path; GP, glass plate; PC, photo-cell; HM, half mirror; Ch, chopper; M, mirror; Att, attenuater; PM, photomultiplier; CRT, cathod ray tube.

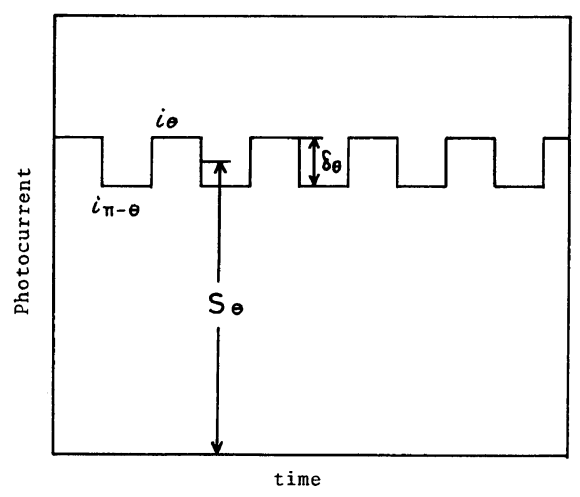

Figure 3. Schematic photocurrent: $\delta_{\theta}$, out-put of lockin amplifier; $S_{\theta}$, averaged photocurrent. 


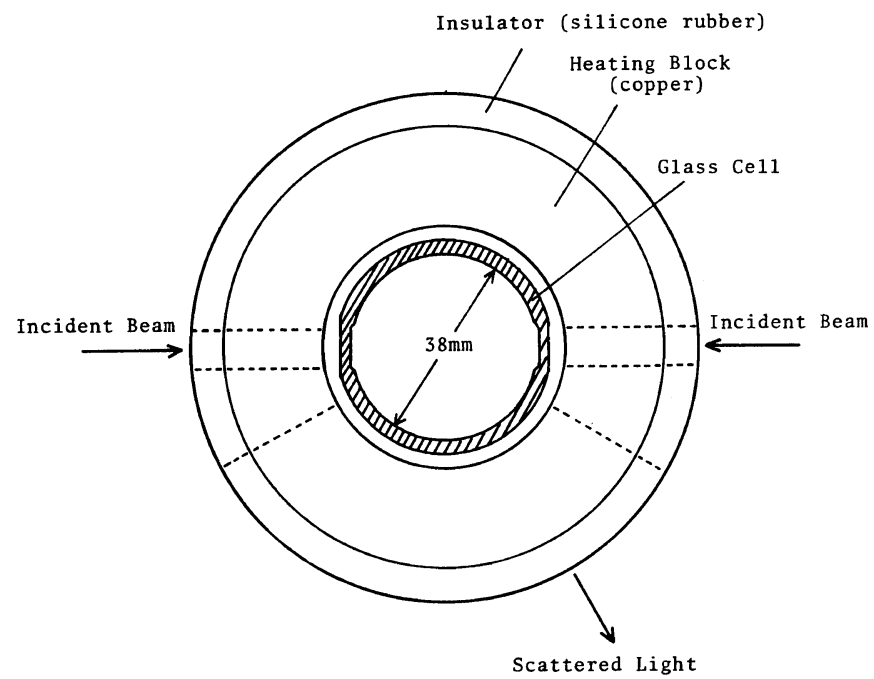

Figure 4. Horizontal cross section of used cell and thermostat.

air cooling $\mathrm{Ar}^{+}$laser (NEC Co., GLG 3000; $488 \mathrm{~nm}-10 \mathrm{~mW}$, vertically polarized) is split into two directions by a half mirror HM. These split beams are introduced into the sample cell alternately from left and right with a chopper $\mathrm{Ch}(225 \mathrm{~Hz})$ and their intensities are equalized with an attenuater Att. The out-put of a photomultiplier PM (1P21) is fed into a lock-in amplifier (NF Circuit Design Block Co., Ltd., Model LI-572B). The frequency and phase of the lock-in amplifier are synchronized with those of the chopper.

Figure 3 shows schematically the signal from PM. The alternate part of the signal $\delta_{\theta}$, which is proportional to $\left(R_{\theta}-R_{\pi-\theta}\right)$, is directly measured with the lock-in amplifier.*1 ${ }^{* 1}$ The averaged intensity $S_{\theta}$, is proportional to $\left(R_{\theta}+R_{\pi-\theta}\right) / 2$, but includes the scattered intensity from the solvent $S_{\theta}{ }^{\circ}$ (an upper suffix ${ }^{\circ}$ denotes solvent). Therefore, the left side of eq 5 is represented by eq 8 ,

$$
\frac{R_{\theta}-R_{\pi-\theta}}{R_{\theta}+R_{\pi-\theta}}=\frac{\delta_{\theta}}{2\left(S_{\theta}-S_{\theta}{ }^{\circ}\right)}
$$

The cross section of a cell and the thermostat is shown in Figure 4. In performance, it is necessary to make some corrections for the asymmetries of the instrument and the cell, and for the Fresnel re-

*1 The direct measurement of $\left(R_{\theta}-R_{\pi-\theta}\right)$ by the lockin amplifier has already been reported by Lee et al. ${ }^{3}$ However, $\theta$ is fixed in their instrument, but changeable in ours.

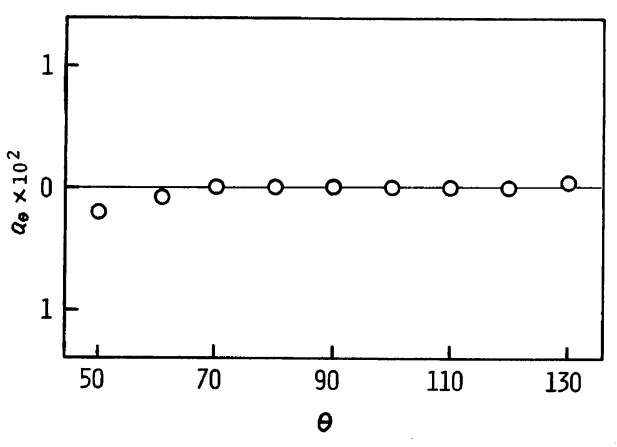

Figure 5. An example of the plot of $a_{\theta} v s . \theta$.

flection on the cell walls.

$\delta_{\theta}{ }^{\circ}$ must be 0 for any solvent, if the symmetry of the instrument is perfect. Let us introduce here the degree of asymmetry of the instrument, $a_{\theta}$, which is defined as

$$
a_{\theta}=\frac{\delta_{\theta}{ }^{\circ}}{S_{\theta}{ }^{\circ}}
$$

In practice, the instrument is adjusted so as to give a value of $a_{\theta}$ as small as possible. This is an essential point in performance. An example of $a_{\theta}$ measured with benzene is shown in Figure 5. Although this example is not the best case, the instrument proved to be easily adjustable to a condition such as to give $a_{\theta}$ of about $10^{-4}$ or less, which is an undetectable order and may be practically neglected. By includ- 
ing the correction factors for this asymmetry and the Fresnel reflection, eq 5 and 8 are modified as

$$
\begin{aligned}
\cos \theta \cdot \rho_{\mathrm{app}} & =1.09_{5} \frac{\delta_{\theta}-a_{\theta} S_{\theta}}{S_{\theta}-S_{\theta}{ }^{\circ}-a_{\theta}\left(\delta_{\theta}-a_{\theta} \delta_{\theta}{ }^{\circ}\right) / 4} \\
& \simeq 1.09_{5} \frac{\delta_{\theta}-a_{\theta} S_{\theta}}{S_{\theta}-S_{\theta}{ }^{\circ}}
\end{aligned}
$$

Further, on including the scattering volume $\left(V_{\theta}\right)$ correction $\left(V_{\pi / 2} / V_{\theta}=f_{\theta} \sin \theta\right)$ for the vertically polarized beam, where $f_{\theta}$ is the cell correction, eq 10 may be replaced by eq 11 , when $R_{\theta}+R_{\pi-\theta} \simeq 2 R_{\pi / 2}$ $\left(\rho_{\text {app }} \ll 1\right)$, so that,

$$
\cot \theta \cdot \rho_{\text {app }}=1.09_{5} \frac{\delta_{\theta}-a_{\theta} S_{\theta}}{S_{\pi / 2}-S_{\pi / 2}^{\dot{ }}} f_{\theta}
$$

Finally, $\rho_{\text {app }}$ is evaluated from the slope at $\theta=\pi / 2$ of the right side of eq $11 \mathrm{vs}$. $\cot \theta$ plot.

The distinctive features of the present instrument can be summerized as follows. Since the scattering intensity difference $\left(R_{\theta}-R_{\pi-\theta}\right)$ is directly measured, an extra signal, coming from a background ${ }^{* 2}$ or stray light admixed with both $R_{\theta}$ and $R_{\pi-\theta}$ signals, nearly cancel out each other, so that the $S / N$ ratio is significantly improved and the measurable range of $\left\langle S^{2}\right\rangle$ is extended below the usual limit. Next, since the numerator and the denominator of the left side of eq 5 are measured simultaneously using the same system, the influence of instability on the light source and other parts is also almost eliminated and thus accuracy is greatly improved. Furthermore, since the present method gives $\rho_{\text {app }}$ using the slope at $\theta=\pi / 2$, there can be avoided the difficulties arising with the usual method on extrapolation to $\theta=0$, as a result of such factors as strong stray light from the cell wall and distortion of the cells in low angle measurements.

An additional merit of our instrument is that a single operation at a certain fixed angle gives $\rho_{\text {app }}$ immediately (see eq 5), and thus rapid processes accompanied by a change in $\left\langle S^{2}\right\rangle$ can be followed. The present instrument is also used as an ordinary single beam photometer, by shutting out one of the incident beams.

*2 The background is comprised primarily of scattering due to the first part in the expansion of $P(\theta)$ (see eq 3 ), and due to the density fluctuation.

\section{EXAMPLES OF EXPERIMENTS}

Experimental results on polystyrene solutions obtained by using the present instrument are as follows. Sample polystyrenes are the so called monodisperse standard polystyrenes prepared by Toyo Soda Manufacturing Co., Ltd. The sample symbols and molecular weights were provided by the supplier. The samples were used without further purification. The solvents, benzene and cyclohexane, were purified by the usual methods. The solvents and solutions were clarified by filtration through a $0.45 \mu \mathrm{m}$ membrane filter (Toyo Roshi Co., Ltd., TM-2P). The $0.1 \mu \mathrm{m}$ and $0.22 \mu \mathrm{m}$ filters were also used for low molecular weight samples, but no change in results was observed.

In Figure $6, \delta_{\theta}$ for F4 $\left(M_{w}, 4.28 \times 10^{4}\right)$ and F20 $\left(M_{w}, 1.86 \times 10^{5}\right)$ in benzene are plotted against $\cot \theta$. The figure shows that the value of $\delta_{\theta}$ can be determined accurately by the lock-in amplifier, even if $\delta_{\theta}$ is small in comparison with $S_{\theta}$; the plots give straight lines passing through the origin. The values of $\rho_{\text {app }}$ evaluated by eq 11 are 0.023 (F4) and 0.137 (F20), respectively.

Figure 7 shows concentration dependences of $\rho_{\text {app }}$ for F4 in benzene and cyclohexane. From the

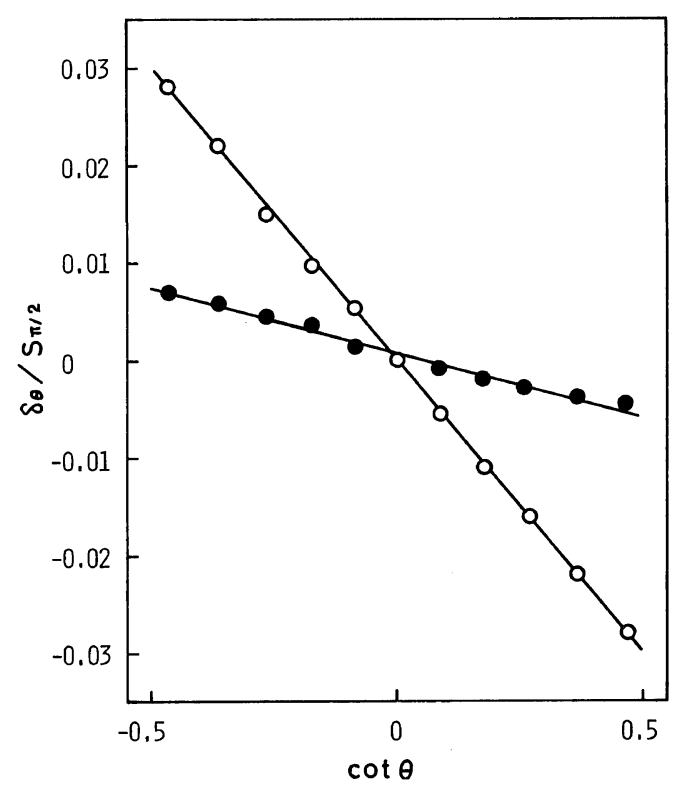

Figure 6. Plots of $\delta_{\theta} / S_{\pi / 2} v s$. $\cot \theta:-, \mathrm{F} 4\left(M_{w}, 4.28 \times\right.$ $\left.10^{4} ; C, 3.09 \times 10^{-3} \mathrm{~g} \mathrm{~cm}^{-3}\right) ;$ O, F20 $\left(M_{w}, 1.86 \times 10^{5}\right.$; $\left.C, 6.80 \times 10^{-4} \mathrm{~g} \mathrm{~cm}^{-3}\right)$, in benzene at $20^{\circ} \mathrm{C}$. 


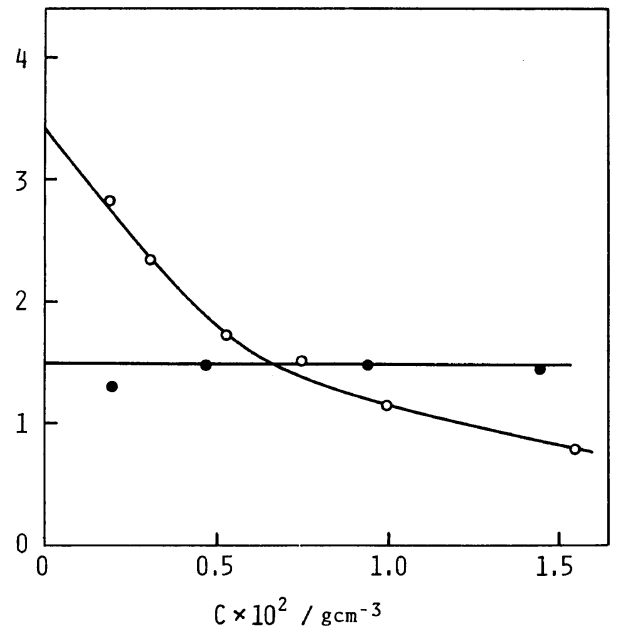

Figure 7. The concentration dependence of $\rho_{\text {app }}$ for F4 $\left(M_{w}, 4.28 \times 10^{4}\right): \bigcirc$, in benzene at $20^{\circ} \mathrm{C} ; \bigcirc$, in cyclohexane at $34.5^{\circ} \mathrm{C}$.

intercept at zero concentration, $7.6 \mathrm{~nm}$ for a benzene solution and $5.8 \mathrm{~nm}$ for a cyclohexane solution were obtained respectively for the values of $\left\langle S^{2}\right\rangle^{1 / 2}$. These values correspond to about $2 \%$ of the wavelength used. As far as we know, these are the smallest values which have been obtained by LS measurements.

It is interesting that these values are in agreement with the extrapolated values from the relations, $\left\langle S^{2}\right\rangle^{1 / 2} \sim M$ in high-molecular-weight regions. ${ }^{4,5}$ Figure 8 shows double logarithmic plots of $\left\langle S^{2}\right\rangle^{1 / 2}$ vs. $M_{w}$ in cyclohexane at $34.5^{\circ} \mathrm{C}$ (the $\Theta$-temperature) and in benzene at $20^{\circ} \mathrm{C}$ for five samples $\left(M_{w}\right.$ : F4, $4.28 \times 10^{4} ; \mathrm{F} 20,1.86 \times 10^{5} ; \mathrm{F} 40,4.22 \times 10^{5} ; \mathrm{F} 80$, $7.75 \times 10^{5} ; \mathrm{F} 126,1.26 \times 10^{6}$ ). The plots are represented by straight lines, giving the following relations (in $\mathrm{nm}^{2}$ ), respectively:

in cyclohexane,

$$
\left\langle S^{2}\right\rangle_{\theta}=8.35 \times 10^{-4} M_{w},
$$

in benzene,

$$
\left\langle S^{2}\right\rangle=2.6 \times 10^{-4} M_{w}^{1.16} .
$$

The former relation is in good agreement with that

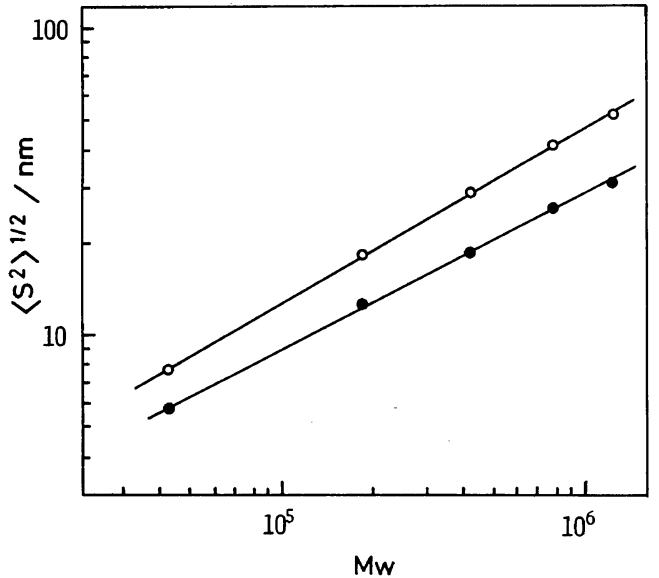

Figure 8. Double logarithmic plots of $\left\langle S^{2}\right\rangle^{1 / 2}$ against $M_{w}: \bigcirc$, in benzene at $20^{\circ} \mathrm{C} ; \bigcirc$, in cyclohexane at $34.5^{\circ} \mathrm{C}$.

of Berry $\left(\left\langle S^{2}\right\rangle=7.75 \times 10^{-4} M_{w} ; M_{w}, 3.94 \times 10^{5} \sim\right.$ $\left.4.4 \times 10^{6}\right)^{4}$ and also with the result of Miyaki et $a l .^{5}$ The latter relation is also in good agreement with that of Fukuda et al. $\left(\left\langle S^{2}\right\rangle=2.3_{1} \times 10^{-4}\right.$ $\left.M_{w}{ }^{1.16} ; M_{w}, 4.9 \times 10^{5} \sim 1.34 \times 10^{7}\right){ }^{6}$

Finite concentration ranges will be considered later.

Acknowledgment. This work was partially supported by a Grant from The Asahi Glass Foundation for the contribution to Industrial Technology, for which the authors are very grateful.

\section{REFERENCES}

1. P. J. Flory, "Statistical Mechanics of Chain Molecules," Interscience, New York, N.Y., 1969, Chapter IX.

2. For example, H. Utiyama, Y. Tsunashima, and $\mathbf{M}$. Kurata, J. Chem. Phys., 55, 3133 (1971).

3. S. P. Lee, W. Tscharnuter, and B. Chu, Rev. Sci. Instrum., 46, 1278 (1975).

4. G. C. Berry, J. Chem. Phys., 44, 4550 (1966).

5. Y. Miyaki, Y. Einaga, and H. Fujita, Macromolecules, 11, 1180 (1978).

6. M. Fukuda, M. Fukutomi, Y. Kato, and T. Hashimoto, J. Polym. Sci., Polym. Phys. Ed., 12, 871 (1974). 